\title{
The effect of verbal training on concept identification in disadvantaged children
}

ELLIN KOFSKY

THE JOHNS HOPKINS UNIVERSITY SCHOOL OF MEDICINE

Training disadvantaged children in labelling and discriminating component stimulus attributes resulted in greater attention to these attributes in inductive concept attainment, but in no greater success in solving concept tasks.

The poor abstracting skills of disadvantaged children (Deutsch, 1965) often have been attributed to their language deficit. Vocabulary enrichment programs have been proposed as a remedy for poor conceptual performance on the assumption that possession of labels would automatically enhance Ss' ability to note and abstract similarities among objects. However, there is considerable debate about whether and under what conditions training in stimulus labelling facilitates subsequent conceptual performance (Tighe \& Tighe, 1966). The following experiment investigated the effect of language training on the concept attainment performance of disadvantaged children. Method

Three groups of 42 lower class Negro kindergartners apiece (mean age $=5.3$ years) differed in familiarity with the concept stimuli and in ability to describe the stimuli. Group $T$ had attended a Head Start Nursery School which provided some lessons in labelling colors, shapes and sizes of familiar objects. They were also trained in small groups for $2015-\mathrm{min}$. sessions to describe the colors, shapes and sizes of stimuli like those in the concept task. Three sets of five stimuli apiece pasted on white cards were used in training. The stimuli in each set varied in one dimension, either color (red, blue, yellow, green, and orange), form (star, cross, circle, square, and triangle) or size (.5-2.5 sq. in.). In each set, Ss were trained to choose the figure which was like the one $E$ held, to find the stimulus $E$ named (e.g., the red or blue), and to name the figure $E$ pointed to. Mistakes were corrected and Ss did not proceed from matching to selecting or from selecting to naming until they performed perfectly with each stimulus. Then another set was presented. The same procedure was also used with figures varying in two dimensions. Group $H$, in a similar Head Start Program, was not specifically trained to describe geometric forms, and a control group (C) had no prior school experience or training.

In order to establish that differences in language skill existed, Ss from all three groups were tested individually on the training stimuli subsequent to the concept task. The three sets of five stimuli described above were presented with the same instructions: to find a match for, select and describe each of the five figures on request. The selection and naming of sizes was restricted to three values. The order of presentation of sets and figures within each set was randomized.

The four concept stimuli were generated from two binary dimensions. For each third of the group, a different dimension was relevant, either color (red vs. blue), size (.3 vs. $.7 \mathrm{sq}$. in.) or form (circle vs. square). The irrelevant dimension was drawn from the remaining two cues. Each $S$ was seated before a screen which had two handles below it. The stimuli were projected successively and $S$ was to try to find out which lever to press for each stimulus, e.g., to press the left for reds and the right for blues. A correct response was rewarded with a marble. Ss were given a sample task to insure that they understood the procedure. The stimulus sequence was controlled for position biases and for stimulus frequency within 16 trial blocks. Criterion was 30 correct responses in 32 trials within 160 trials.

\section{Results}

Mean errors made in describing and discriminating the training stimuli are presented in Table 1. An analysis of variance revealed significant training effects $(F=11.57, \mathrm{df}=2 / 120, \mathrm{p}<.001)$, and an interaction of training and task $(F=6.34, d f=4 / 240, p<.001)$. Although Ss did not differ in their ability to match stimuli, the $T$ group knew and could respond to more color, shape and size names than the other two groups. An analysis of just those stimulus values used in the concept tasks (red, blue, large, small, circle, and square) produced similar results: superior linguistic performance by $T$ Ss $(F=7.57, d f=2 / 120, p<.001)$.

Although the $T$ group was more proficient in labelling all aspects of the stimuli, relevant and irrelevant, they were not more proficient in concept identification. The mean errors for the $\mathrm{T}, \mathrm{H}$ and $\mathrm{C}$ groups were 58.2, 57.1, and 62.1 respectively. Variations in the relevant dimension did not affect performance.

Errors scores reflect S's response to relevant stimulus attributes. Yet young Ss often respond perseveratively to irrelevant stimulus cues (Osler \& Kofsky, 1965). Perseveration was detected by count-

Table 1. Mean Errors on Perceptual Tasks

\begin{tabular}{lccc} 
Group & Motching & $\begin{array}{c}\text { Tasks } \\
\text { Selecting }\end{array}$ & Noming \\
\hline$T$ & .64 & 2.07 & 1.90 \\
$H$ & .93 & 3.43 & 3.64 \\
$C$ & 1.17 & 4.11 & 5.14 \\
\hline
\end{tabular}


ing separately for each dimension, regardless of relevance, the number of times $S$ pressed left and right for each value, e.g., for reds and blues. From these data, the amount of information transmitted from each dimension to $S$ 's response and the significance level for each transmission were calculated (Attneave, 1959; Osler \& Kofsky, 1965). Analyses showed that about the same number of Ss in each group (16 T Ss, $17 \mathrm{H}$ Ss and $13 \mathrm{C} \mathrm{Ss}$ ) significantly attended to relevant cues, but more trained $\mathrm{Ss}_{2} 31$, responded to any stimulus cue, regardless of relevance, than $S s$ in the $H(23)$ or $C(23)$ groups $(z=2.08$, $\mathrm{p}<.05)$. Thus training enabled more $\mathrm{Ss}$ to attend to stimulus attributes but trained Ss did not reach criterion more frequently or faster than untrained Ss. Discussion

Several explanations may account for the ineffectiveness of training. Even if stimulus labelling facilitates discrimination performance, the effects of language training might be negligible. Possession of a label does not guarantee appropriate use of it to mediate learning. Young Ss, particularly, may not profit from vocabulary enrichment because they haven't learned how to use words to guide performance (Kendler \& Kendler, 1962). Conversely, when E supplies labels to an experimental group, there is no guarantee that a control group has not manufactured labels of its own. Perhaps the important feature to label is not the stimulus value but connections between responses and rewards. Verbal inductive rules may provide better mediators than stimulus labels since concept identification requires induction as well as discrimination of relevant stimulus attributes. Evidence from this study suggests this is the case, since the $T$ group had a larger stimulus vocabulary and paid more attention to stimulus attributes, but they were not more successful in isolating or inferring the relevant cue.

\section{References}

Attneave, F. Applications of information theory to psychology. New York: Holt, 1959.

Deutsch, M. The role of social class in language development and cognition. Amer. J. Orthopsychiat., 1965, 35, 78-88.

Kendler, H. H., \& Kendler, T. S. Vertical and horizontal processes in problem solving. Psychol. Rev., 1962, 69, 1-16.

Osler, S. F., \& Kofsky, E. Stimulus uncertainty as a variable in the development of conceptual ability. J. exp. child Psychol., $1965,2,264-279$.

Tighe, L. S., \& Tighe, T. J. Discrimination learning: two views in historical perspective. Psychol. Bull., 1966, 66, 353-370. Note

1. This study is part of a continuing project supported by NIH (HD 00754), the Office of Economic Opportunity (Contract No. 510) and the Grant Foundation. The aid of N. Caldwell, M. Wasserman, $P$. Morrissey and P. Schaeffer in tutoring and testing Ss and of the Baltimore City Public School system for making Ss available was greatly appreciated. 